\title{
A Minimum Discrimination Information Control Chart for Stationary Autocorrelated Processes
}

\author{
Pairoj Khawsithiwong ${ }^{1}$ and Nihal Yatawara ${ }^{2}$ \\ 1 Department of Statistics, Faculty of Science, Silpakorn University, \\ Nakorn Phatom, Thailand. E-mail: pairoj@su.ac.th \\ ${ }^{2}$ Department of Mathematics and Statistics, Curtin University of \\ Technology, Perth, Western Australia. E-mail: n.yatawara@curtin.edu.au
}

\begin{abstract}
A procedure for simultaneous monitoring of both the process mean and the standard deviation with a single control chart based on the Kullback-Leibler $(K L)$ statistic is proposed for stationary Gaussian autocorrelated processes. The residual based MDI control chart is developed and then the performance is investigated through Monte Carlo simulations in situations of sustained shifts using the average run length (ARL) as the preferred criterion for comparisons. The results show that the proposed chart performs exceptionally well for detecting shifts in process mean even when standard deviation changes.
\end{abstract}

Key Words: Simultaneous monitoring, Autocorrelated processes, Residual control chart, Kullback-Leibler function, Exponentially weighted moving average.

\section{Introduction}

As discussed by Gan [1], a practical simultaneous monitoring procedure requires a pair of control charts with two-sided control limits for mean and standard deviation which are concurrently implemented to detect changes in a process caused by special causes of variability. In addition, Gan [2] argued that a change in process variability affects both the mean chart and the standard deviation chart in a combined scheme. Consequently, the use of a single control charting procedure is advocated as an alternative way to deal with this problem.

To monitor autocorrelated processes, two general approaches are introduced. The first method is based on an effort to modify control limits of 
the traditional control charts by taking the effect of autocorrelation into account (e.g. see Vasilopoulos and Stamboulis [3], Runger [4], Kramer and Schmid [5], Zhang [6], Jiang, Tsui, and Woodall [7], Atienza, Tang and Ang [8] and references there in). The other attempts to transform the original data to uncorrelated data by fitting time series models and then monitoring a process through one-step ahead forecast errors (residuals) (e.g. see Alwan and Roberts [9], Harris and Ross [10], Montgomery and Mastrangelo [11], Wardell, Moskowitz, and Plante [12], Testik [13] and references there in).

In this research, the well known Kullback-Leibler function is used for establishing a minimum discrimination information (MDI) control function which gives a minimum distance between distributions of monitoring state and a reference state. Since the MDI control function is expressible in a simple form as a linear combination of effects due to all parameters, it is used as the primary tool for constructing a simultaneous control chart involving residuals from univariate autocorrelated processes. Finally, its performance under various scenarios is also investigated.

\section{Minimum Discrimination Information}

Kullback [14] introduced the MDI principle as a fundamental principle in statistics that provides a unified method to handle several problems in statistical inference associated with hypothesis testing, estimation, regression analysis, multivariate techniques, and analysis of contingency tables. This principle associated with a quantity that measures the information discrepancy or divergence or distance between two probability distributions is defined by

$$
K L\left(f_{(1)}: f_{(0)}\right)=\int \log \frac{f_{(1)}(y)}{f_{(0)}(y)} d F_{(1)}(y) \geq 0
$$

where $f_{(i)}(y)=d F_{(i)}(y), i=0,1$, are probability densities. The values of the $K L$ statistic are generally greater than zero with equality if and only if both distributions are the same. Since the $K L$ statistic gives a measure of uncertainty or entropy of the probability distribution, $F_{(1)}(y)$, relative to the reference probability distribution, $F_{(0)}(y)$, it is also named as relative entropy or cross entropy. The relative entropy is a convex and an asymmetric function. For samples of size $n$, the $2 n$ times the relative entropy is asymptotically distributed as a chi-square, $\chi^{2}$, random variable, 
with $K$ degrees of freedom where $K$ is the number of model parameters.

\section{Residual Based MDI Control Chart}

Suppose the predictive probability distributions of reference and monitoring state forecasts are Gaussian with mean $\hat{Y}_{(i) t}$ and variance $\sigma_{(i)}^{2}, i=0,1$, where the values of subscript $i=0,1$ indicate the in-control and the monitoring state, respectively. The predictive density corresponding to both states can be expressed as

$$
f_{(i)}\left(y_{t} \mid y_{t-1}, \ldots, y_{1}\right)=\frac{1}{\sqrt{2 \pi \sigma_{(i)}^{2}}} \exp \left(-\frac{1}{2 \sigma_{(i)}^{2}}\left(y_{t}-\hat{y}_{(i) t}\right)^{2}\right), \quad i=0,1
$$

Accordingly, the $K L$ statistic, the minimum distance between such states is given by

$$
K L\left(f_{(1)}: f_{(0)}\right)=\frac{1}{2}\left(\frac{\sigma_{(1)}^{2}}{\sigma_{(0)}^{2}}-\log \frac{\sigma_{(1)}^{2}}{\sigma_{(0)}^{2}}-1\right)+\frac{1}{2} \frac{\left(\hat{y}_{(1) t}-\hat{y}_{(0) t}\right)^{2}}{\sigma_{(0)}^{2}}
$$

Define the forecasts based on all known model parameters from an $A R M A(p, q)$ process as

$$
\hat{y}_{(0) t}=\mu_{(0)}+\sum_{j=1}^{p} \phi_{(0) j}\left(y_{t-j}-\mu_{(0)}\right)+\sum_{l=1}^{q} \theta_{(0) t}\left(y_{t-l}-\hat{y}_{(0) t-l}\right)
$$

and denote the residuals by $e_{(0) t}=y_{t}-\hat{y}_{(0) t}$. As shown in Wardell et al. [12], the magnitude of change in a process mean and in a process variance can be estimated by the mean and the variance of $e_{(0) t}$, respectively. Also, it can be argued that, in the monitoring period, the quantity $\hat{y}_{(1) t}-\hat{y}_{(0) t}$ giving the magnitude of shift in a process mean is equivalent to the mean of $e_{(0) t}$. Substituting this in equation (3) and multiply by $2 n$, yields the residual based MDI control function which can be formulated in terms of the mean and the variance of $e_{(0) t}$ as

$$
I_{R}=n\left(\frac{\sigma_{e_{(0)}}^{2}}{\sigma_{(0)}^{2}}-\log \frac{\sigma_{e_{(0)}}^{2}}{\sigma_{(0)}^{2}}-1\right)+n \frac{\mu_{e_{(0)}}^{2}}{\sigma_{(0)}^{2}}
$$


where $\mu_{e_{(0)}}$ and $\sigma_{e_{(0)}}^{2}$ are the mean and the variance of residuals and $n$ is the number of residuals used for calculations. Clearly, the $I_{R}$ control function behaves as an asymptotic $\chi^{2}$ random variable with 2 degrees of freedom.

To implement the $I_{R}$ control function, it is required to estimate a local mean and a variance of the residuals $e_{(0) t}$. With these estimates, the control function can be rewritten as

$$
I R_{t}=n\left(\frac{\hat{\sigma}_{t}^{2}}{\sigma_{(0)}^{2}}-\log \frac{\hat{\sigma}_{t}^{2}}{\sigma_{(0)}^{2}}-1\right)+n \frac{\bar{e}_{t}^{2}}{\sigma_{(0)}^{2}}
$$

where $\bar{e}_{t}$ and $\hat{\sigma}_{t}^{2}$ are respectively an estimated local mean and an estimated variance at time $t$ of the residuals. For on-line parameter estimation, the double exponentially weighted moving average (DEWMA) technique proposed by Sweet [15], is considered. The DEWMA consists of the exponentially weighted moving average (EWMA) and the exponentially weighted moving variance (EWMV), defined respectively by

$$
\bar{e}_{t}=\lambda_{1} e_{(0) t}+\left(1-\lambda_{1}\right) \bar{e}_{t-1}, 0<\lambda_{1} \leq 1
$$

and

$$
\hat{\sigma}_{t}^{2}=\lambda_{2}\left(e_{(0) t}-\bar{e}_{t-1}\right)^{2}+\left(1-\lambda_{2}\right) \hat{\sigma}_{t-1}^{2}, 0<\lambda_{2} \leq 1
$$

where $\lambda_{1}$ and $\lambda_{2}$ are smoothing parameters. Without loss of generality, both smoothing parameters can be made equal, $\lambda_{1}=\lambda_{2}=\lambda$, for a simple design of the control chart. The values of $\lambda$ are selected to obtain a smoothed local mean and a variance, respectively. In practice, the values of such smoothing parameters are recommended to be in the interval $0.05 \leq \lambda \leq 0.25$, see Montgomery (2001). To establish the control function, the approximate number of observations required to estimate the parameters in equation (6) is given by $n=\frac{2-\lambda}{\lambda}$. An out of control is signalled if $I R_{t}>h_{I R}$ where the $h_{I R}$ is a percentage point from the asymptotic $\chi^{2}$ distribution with 2 degrees of freedom.

Figure 1 depicts the behavior of $\operatorname{IR}(E W M A)$ control chart for monitoring a process in various situations of changes in process mean and/or variability. The $\operatorname{IR}(E W M A)$ control chart seems to be capable of detecting changes in all situations as shown in Figures 1(b) to 1(f), particularly, for detecting shifts in mean. This is mainly because the residuals $e_{(0) t}$ contain the effects of all changes in process parameters. 


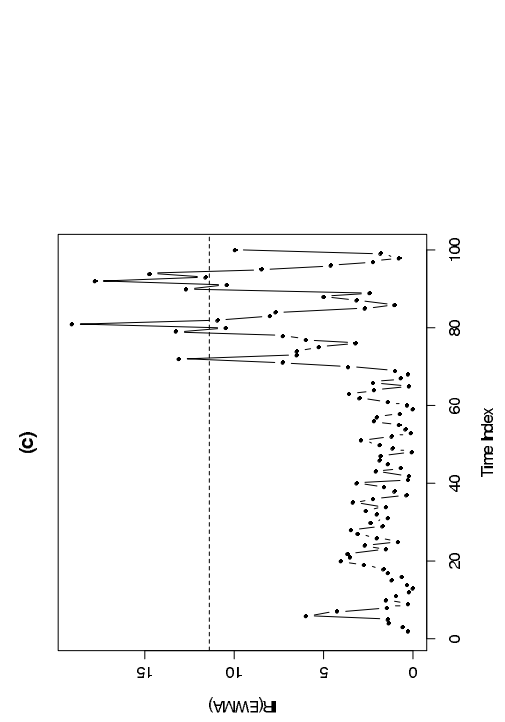

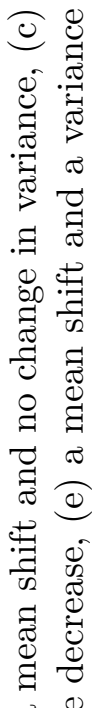
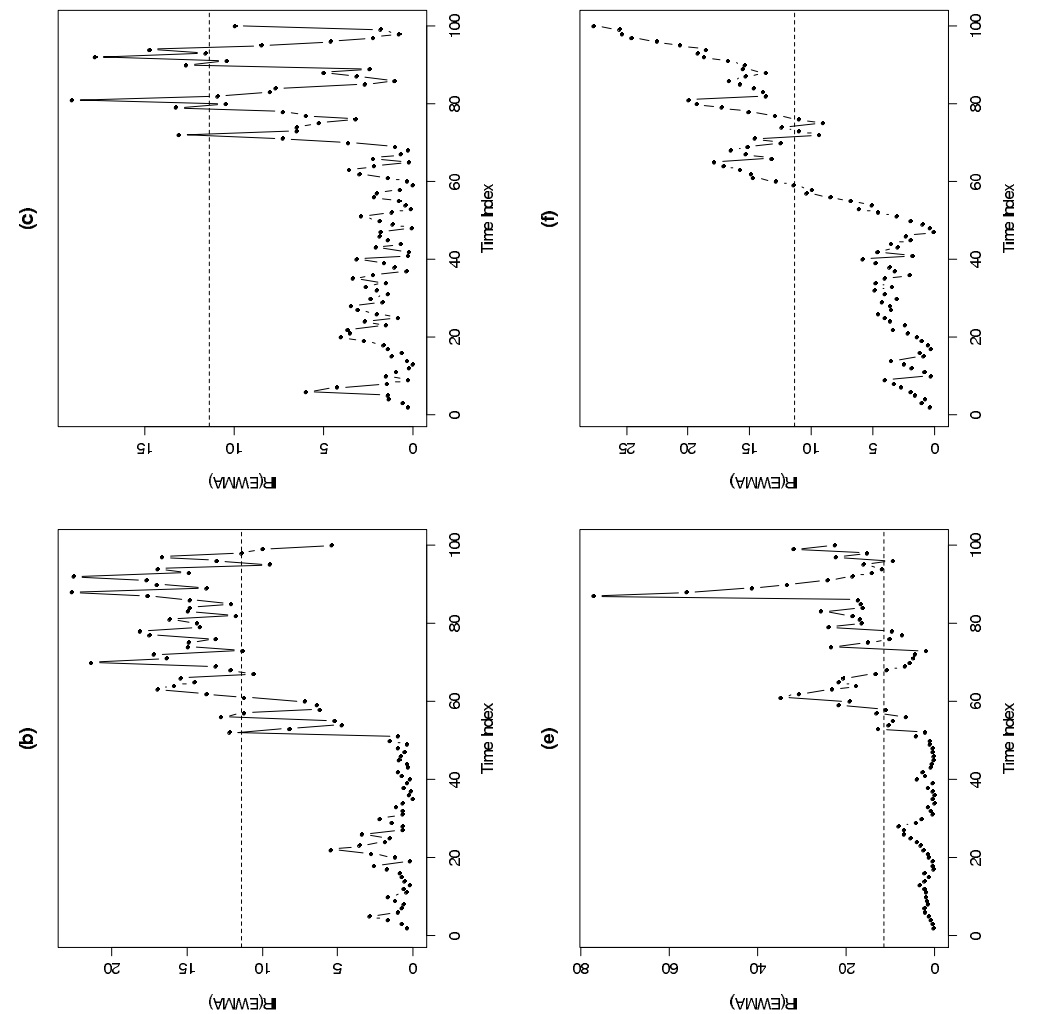
กิ

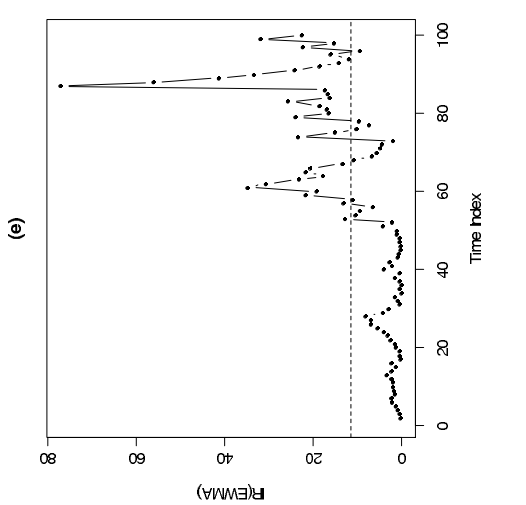

e

i

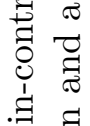

(ల)

is .

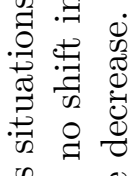
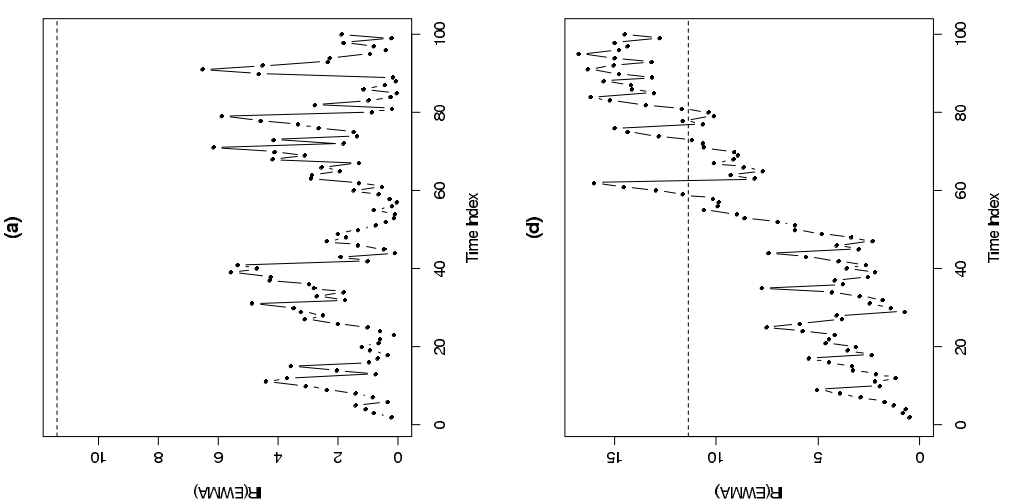

응

家 0

- ठ

.

苛

过

$\mathcal{\nabla} \cdot \stackrel{\sigma}{\sigma}$

$\sum \stackrel{5}{>}$

से

둥

Ð

.. .

$\ddot{0} ّ$

当 寻

的 


\section{Performance Study}

A performance comparison between the residual based MDI control chart and the corresponding combined (EWMA, EWMV) control charting techniques is under taken utilizing $\operatorname{ARMA}(1,1)$ processes where model parameters are given by $\left(\phi_{1}, \theta_{1}\right)=(0.3,-0.2),(-0.3,0.2),(0.3,-0.8),(-0.3,0.8)$, $(0.8,0.5),(0.8,-0.4),(0.9,0.7)$, and $(0.8,-0.8)$. Assume that the model parameters in the monitoring period are the same as those in the in-control state.

Let $\mu_{(0)}$ and $\sigma_{(0)}^{2}$ be the mean and the variance of white noise in the incontrol state. The process attributes are set up for the periods of monitoring with the mean and the standard deviation obtained from the expressions, $\delta_{M}=\frac{\mu_{(1)}-\mu_{(0)}}{\sigma_{(0)}}$ and $\delta_{S D}=\frac{\sigma_{(1)}}{\sigma_{(0)}}$ where $\delta_{M}$ and $\delta_{S D}$ are the magnitudes of shifts in process mean and standard deviation from the in-control state. In this study, the $\delta_{M}$ is varied between $0,0.25,0.5,0.75,1,2$, and 3 and $\delta_{S D}$ assumes values $0.25,0.5,0.8,1,1.1,1.2$, and 2 and all possible combinations of $\delta_{M}$ and $\delta_{S D}$ in this set are considered. The simulations are conducted only for $\delta_{M}>0$, since the ARL results will be similar when $\delta_{M}<0$.

To make the control charts comparable, the control charting scenarios are adjusted to the same in-control false alarm rate of 0.002 . This equivalently gives an in-control ARL value of 500. Also, for each chart in the combined control charting scheme, the in-control false alarm rate was set to be 0.001 . The ARL values were obtained from 10,000 simulations for series of length 50. Moreover, for parameter updating with exponential smoothing techniques, the required smoothing constants for mean and variance are both set to be 0.2 . The control limits in the proposed MDI control chart are obtained from 50 generated data in the in-control period for a specified in-control false alarm rate.

\subsection{Run Length Analysis}

Consider six positively autocorrelated processes; $\left(\phi_{1}, \theta_{1}\right)=(0.3,-0.2),(0.3$, $-0.8),(0.8,0.5),(0.8,-0.4),(0.9,0.7)$, and $(0.8,-0.8)$ having the first-lag autocorrelation $\rho_{1}=0.46,0.64,0.40,0.88,0.32$, and 0.9 and two negatively autocorrelated processes; $\left(\phi_{1}, \theta_{1}\right)=(-0.3,0.2)$ and $(-0.3,0.8)$ with $\rho_{1}=-$ 0.46 and -0.64 , respectively. As shown in Table 1, the $\operatorname{IR}(E W M A)$ control chart shows superior power for detecting shifts in process mean even when 
the standard deviation changes. However, when the process exhibits positive autocorrelation, the combined scheme is more sensitive to detect small shifts in mean when standard deviation increases and the $\operatorname{IR}(E W M A)$ control chart loses its power as indicated by an increase in ARL values.

For positive autocorrelation, detection capabilities of the control charting schemes are affected by autocorrelation. The ARL values from negatively autocorrelated processes decrease more rapidly than those in the positive cases. This is because residuals from such processes become large when a process mean shifts, caused by one-step ahead forecasts moving in opposite direction to that of the mean shift, see Wardell et al. [12].

Detection capabilities of the control charts for mean shifts are mainly affected by the presence of large values of positive $A R M A$ coefficients and a decreasing standard deviation. The impact of autocorrelation on the $\operatorname{IR}(E W M A)$ control chart is rarely a threat to its implementation because it depends on the in-control state residuals that are independent. A significant improvement of these control charts can be obtained by using smaller exponential weights.

\section{Conclusions}

The MDI control function based on the $K L$ statistic giving a minimum divergence between two distributions is developed to discriminate between an in-control and an out-of-control situation in stationary Gaussian autocorrelated processes. This control function includes linear combinations of all the effects of process parameters. Thus, it can be conveniently used to sequentially and simultaneously detect shifts in all process attributes, although its performance is not superior to combined schemes under several different scenarios. In the implementation of the MDI control chart, values of the control function are plotted with a single control limit.

As an alternative view, the $I_{R}$ control function measures the distance between the distributions of the reference state and the monitoring state residuals. The distribution of the residuals $e_{(0) t}$ is assumed to be distributed as Gaussian with mean zero and variance $\sigma_{(0)}^{2}$, in the reference state and distributed as Gaussian with mean $\mu_{e_{(0)}}=\sum_{k=0}^{\infty} \omega_{k}\left(\mu_{(1)}-\mu_{(0)}\right)$ and variance $\sigma_{(1)}^{2}$ where $\omega_{k}$ is a function of time series coefficients as defined in Wardell et al. [12], in the monitoring state. 


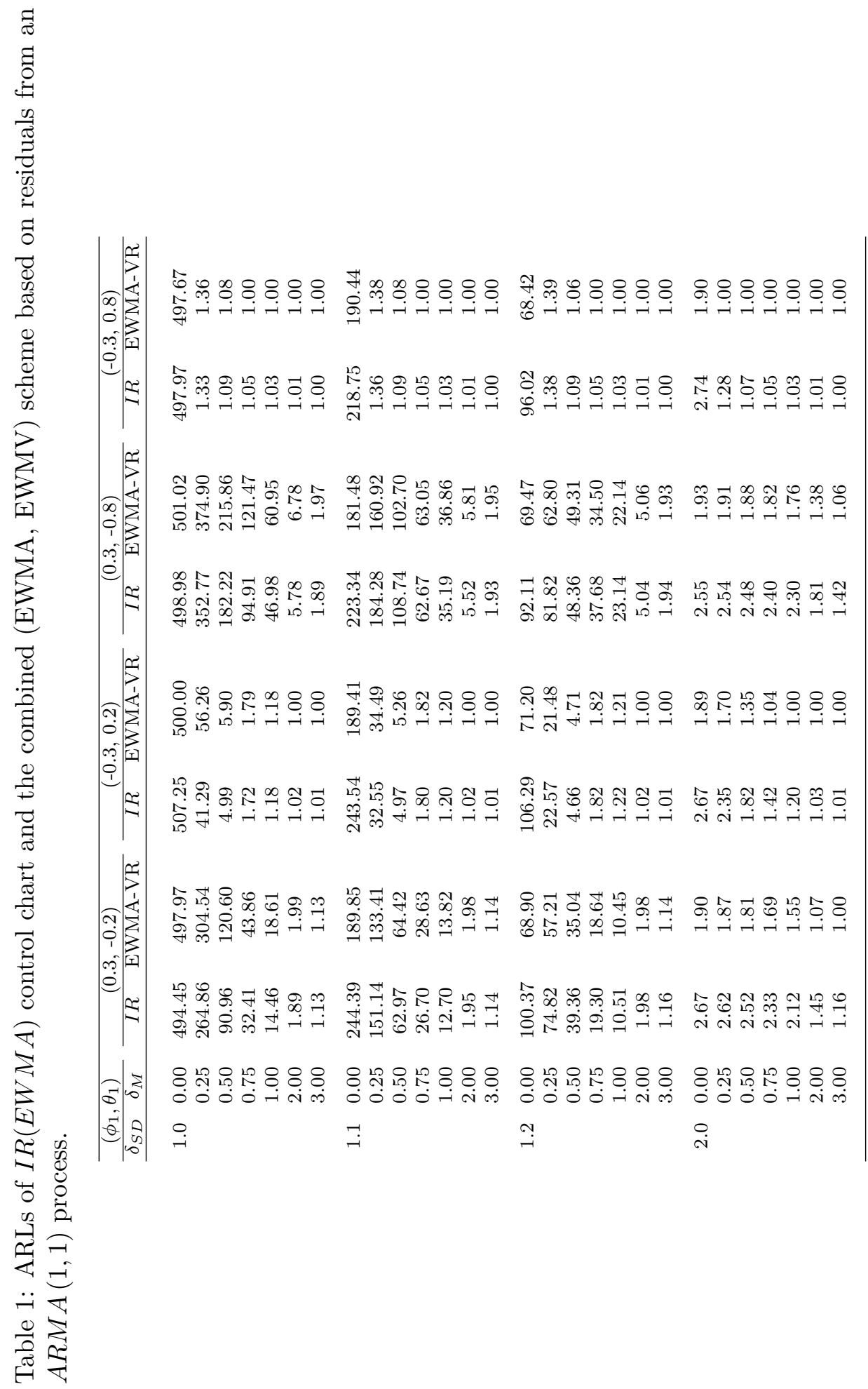




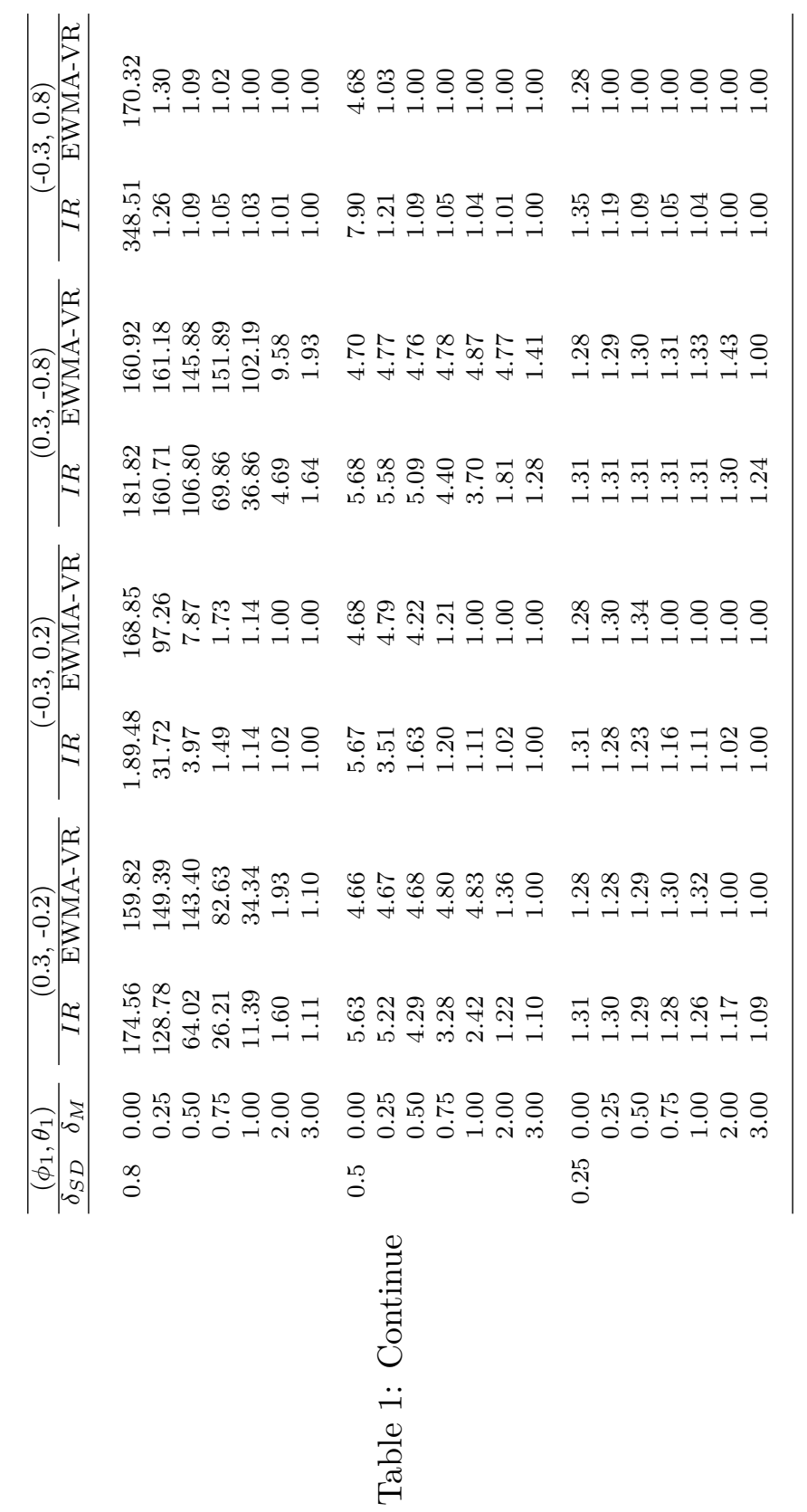




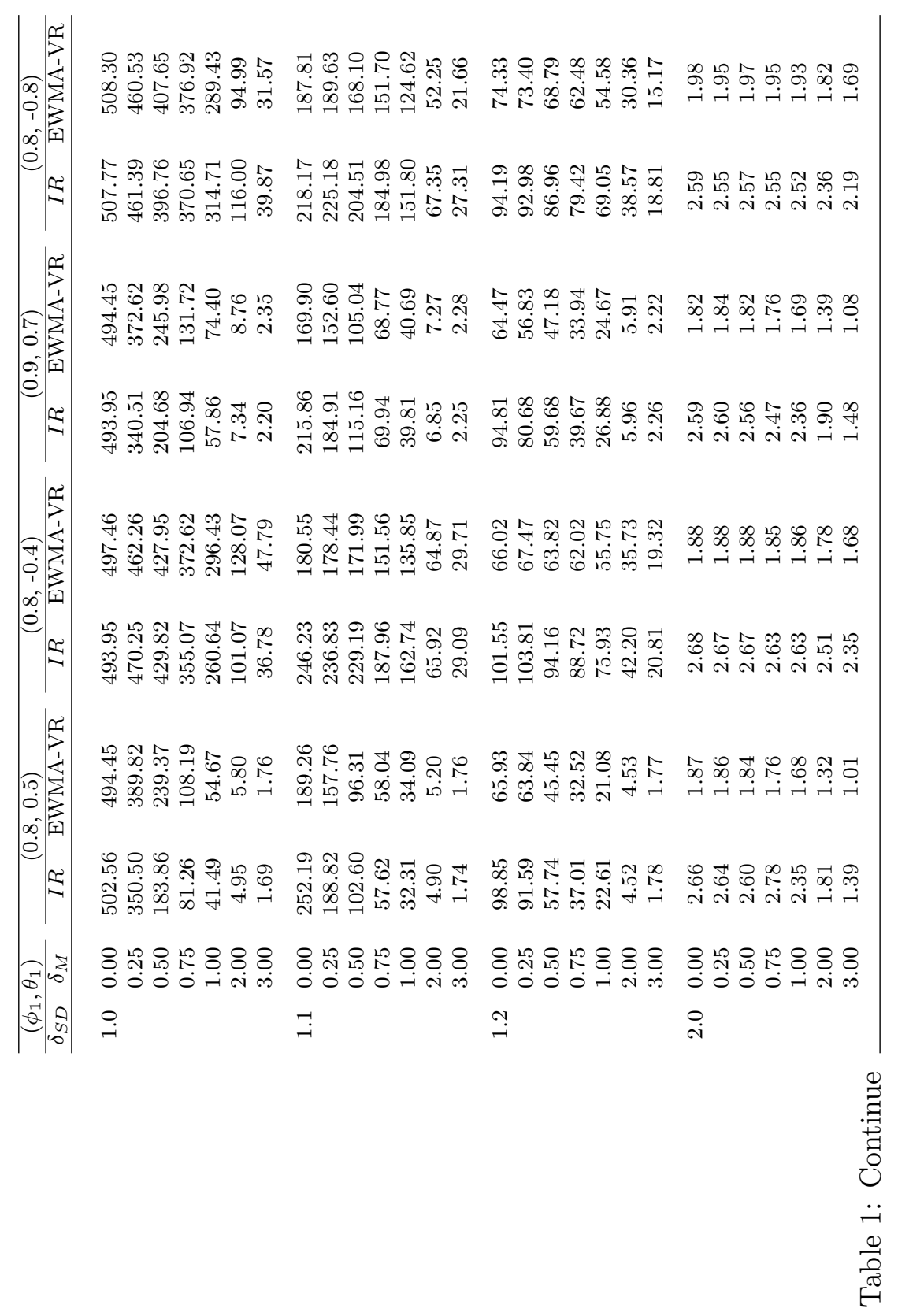




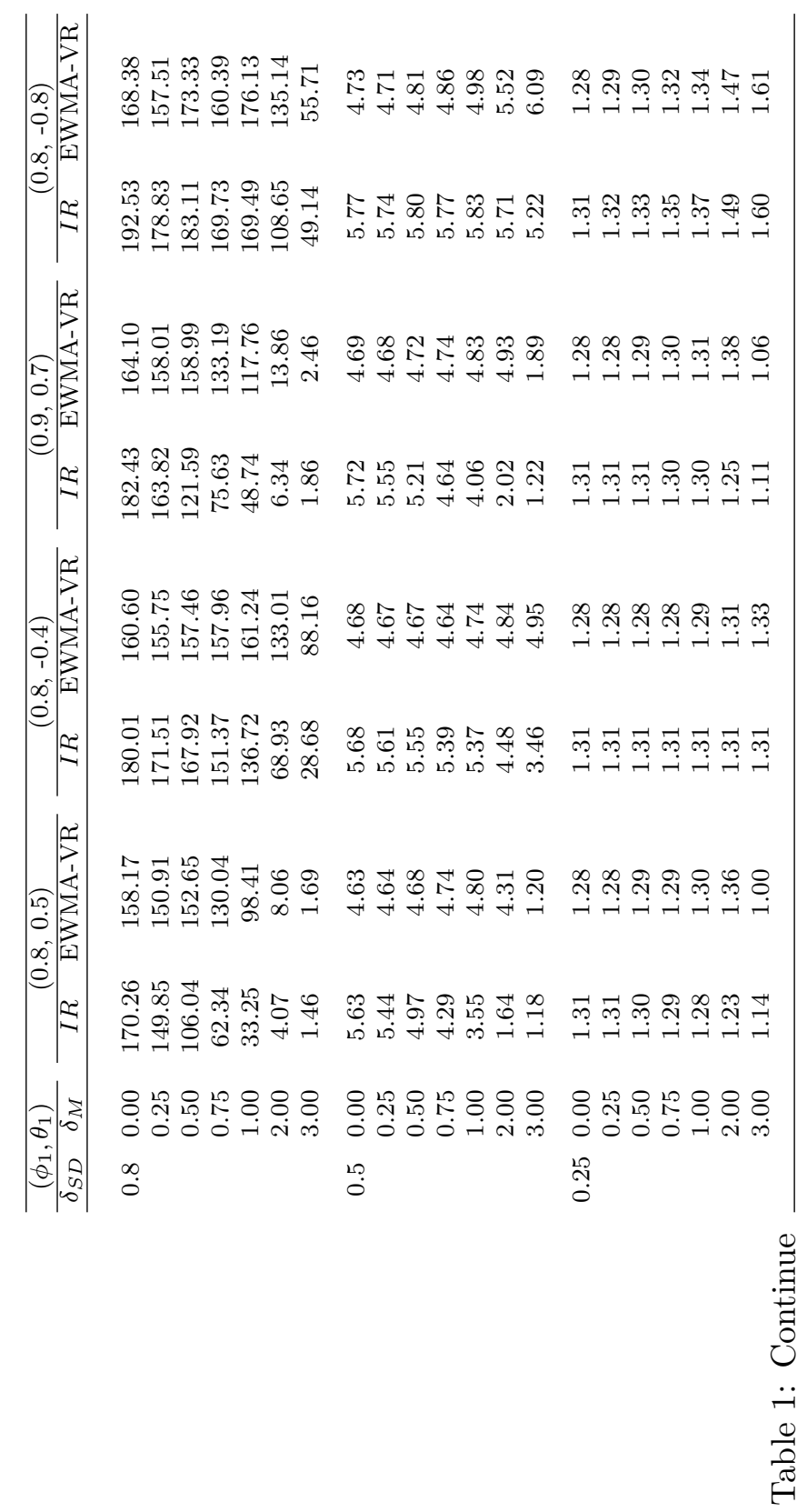


The residual based MDI control chart is widely applicable. Results from Monte Carlo simulations show its outstanding performance for detecting shifts in process mean even when standard deviation changes. Although, it does not perform very well for detecting standard deviation changes, its detection ability is seemingly competitive to those of the combined schemes.

It was identified that the factors mainly affecting the performance of the control charts are the autocorrelation, changes in standard deviation, and the parameter estimates. To improve the detection power of the control charts, smaller smoothing constants at the parameter estimation stage may be used.

A further concern dealing with the MDI control function is its performance when time series coefficients are subject to change. In this case, the process variability changes, since the process variability is a function of white noise variance and the time series coefficients. Wardell et al. (1994) has also discussed the poor performance of the residual control chart for mean detection when $A R M A$ coefficients are subject to change. This is because, when the process mean shifts, the ARMA coefficients get weighed by the magnitude of the mean shifts. It would be interesting to examine the tolerance of the chart to coefficient changes before they begin to influence the ability of the control chart. 


\section{References}

1. F. F. Gan. Joint monitoring of process mean and variance using exponentially weighted moving average control charts. Technometrics, 37(4):446-453, 1995.

2. F. F. Gan. Joint monitoring of process mean and variance. Nonlinear Analysis, 30(7):4017-4024, 1997.

3. A. V. Vasilopoulos and A. P. Stamboulis. Modification of control chart limits in the presence of data correlation. Journal of Quality Technology, 10(1):20-30, 1978.

4. G. C. Runger. Model-based and model-free control of autocorrelated processes. Journal of Quality Technology, 27(4):283-292, 1995.

5. H. Kramer and W. Schmid. Control charts for time series. Nonlinear Analysis, 30(7):4007-4016, 1997.

6. N. F. Zhang. A staistical control chart for stationary process data. Technometrics, 40(1):24-38, 1998.

7. W. Jiang, K.L. Tsui, and W. H. Woodall. A new spc monitoring method: The arma chart. Technometrics, 42(4):399-410, (2000).

8. O. O. Atienza, L. C. Tang, and B. W. Ang. A cusum scheme for autocorrelated observations. Journal of Quality Technology, 34(2):187-199, 2002 .

9. L. C. Alwan and H. V. Roberts. Time-series modeling for statistical process control. Journal of Business \& Economic Statistics, 6(1):87-95, 1988.

10. T. J. Harris and W. H. Ross. Statistical process control procedures for correlated observations. The Canadian Journal of Chemical Engineering, 69:48-57, 1991.

11. D. C. Montgomery and C. M. Mastrangelo. Some statistical process control methods for autocorrelated data. Journal of Quality Technology, 23(3):179-193, 1991. 
12. D. G. Wardell, H. Moskowitz, and R. D. Plante. Run-length distributions of special-cause control charts for correlated processes. Technometrics, 36(1):3-17, 1994.

13. M. C. Testik. Model inadequacy and residuals control charts for autocorrelated processes. Quality and Reliability Engineering International, 21:115-130, 2005.

14. S. Kullback. Information Theory and Statistics. John Wiley \& Sons: New York, 1959.

15. A. L Sweet. Control chart using coupled exponentially weighted moving averages. IIE Transactions, (18):26-33, 1986. 
